How to reference this article

Jeż, T. (2021). Aspetti semioforici della Bibliotheca Sartoriana. Italica Wratislaviensia, 12(1), 107-121.

DOI: http://dx.doi.org/10.15804/IW.2021.12.1.06

\author{
Tomasz Jeż \\ Uniwersytet Warszawski, Polonia \\ tomasz.jez@uw.edu.pl \\ ORCID: 0000-0002-7419-3672
}

\title{
ASPETTI SEMIOFORICI DELLA BIBLIOTHECA SARTORIANA
}

\section{SEMIOPHORIC ASPECTS OF THE BIBLIOTHECA SARTORIANA}

\begin{abstract}
The Wrocław patrician Thomas Rehdiger (1540-1576), during his Wanderjahre, amassed a rich collection of books, manuscripts, coins, precious stones, and works of art. In his will, he bequeathed this collection - gathered mainly during his stays in Padua, Bologna, and Venice - to his hometown, thus giving rise to the first public library in the Wrocław metropolis. In 1589, the Bibliotheca Rhedigeriana was established in the Auditorium Theologicum of St. Elizabeth's Church. In his youth, Rehdiger was educated in the school operating at the church; almost 100 years later, the school's teacher, Daniel Sartorius (1612-1671), gathered a large collection of music prints, which were published in that period in Venetian publishing houses. This unique collection, stored together along with the Rhedigeriana Library, was later merged with the library's content.

What was the significance of this collection in the culture of $17^{\text {th }}$-century Wrocław? This collection, undoubtedly, was assembled based upon inspiration from the Humanistic period, which had been promoted in that Elizabethan school at least as of the times of Laurentius Corvinus. It was supposed to serve the teaching at the Elisabethanum and shape the artistic tastes of its students, who performed state-of-the-art Italian music during school activities or afternoon recess. Attempts were made to introduce the carefully collected repertoire into the liturgical practice of a nearby church, using the technique of contrafactum that was popular at the time. The Sartorian Library's semiophoric function, however, was to define a new form of cultural memory for the Wrocław elites and to outline the original profile of their identity, which was oriented by the same ideals that guided the composers of the Italian Seicento.
\end{abstract}

Keywords: Wrocław, Italian Seicento, music collection, St. Elizabeth's church, Daniel Sartorius 
Thomas Rehdiger (1540-1576) proveniva da una ricca famiglia di 1 cittadini breslaviani i quali, grazie ai proventi derivanti dal commercio e ad opportune unioni matrimoniali, si erano ritrovati nella ristretta cerchia del patriziato di Breslavia (Wachler, 1828, pp. 3-5). La sua ascesa sociale fu suggellata con gli studi intrapresi presso il ginnasio maschile di Santa Elisabetta, che era un centro importante per l'educazione umanistica fin dai tempi di Laurentius Corvinus (Bauch, 1896, p. 128). Dopo il ginnasio, il giovane patrizio fu mandato a studiare a Wittenberg dove, grazie alla protezione di Johann Krafft von Krafftheim, venne affidato alla guida didattica di Filippo Melantone (Wachler, 1828, p. 7). Di lì a breve per Rehdiger iniziò il grand tour europeo, le cui tappe furono Parigi, Orléans, Anversa, Lovanio, Bourges, Valence e poi di nuovo Parigi, da dove si spostò a Padova. Per un po' di tempo restò nell'università padovana, compiendo tuttavia viaggi anche a Bologna, Roma, Napoli e Venezia, dove raccolse per la sua collezione libri, manoscritti, monete, quadri e manufatti di artigianato artistico. Nel 1569 andò ad Anversa e poi a Colonia, dove restò vittima di un incidente e morì. Fece in tempo tuttavia a scrivere il testamento, in cui destinava tutta la sua collezione alla propria città natale, dando così vita alla prima biblioteca pubblica di Breslavia:

Libros meos, quos ego magno labore et sumptu conquisivi, una cum Numismatibus aureis, argenteis, aeneis, statuis, picturis, et reliquis Bibliothecae ornamentis minime vulgaribus cupio et volo quam diligentissime Vratislaviae in uno Conclavi ad id a praedictis duobus heredibus instructo adservari et omnia in eo suis locis distincte et ordinate disponi: ut illa Bibliotheca cum suis ornamentis non tantum Rhedigerianae Familiae (penes quam perpetuo esse volo) honori, verum etiam aliis usui et voluptati esse possit. (Wachler, 1828, pp. 70-71)

Nella sua forma originale la raccolta di Thomas Rehdiger mostrava chiaramente i tratti della personalità del collezionista ed il suo fascino per 1'antico, il Rinascimento e la Riforma (Houszka, 1998, p. 14). Questa collezione straordinariamente ricca, che dai contemporanei fu stimata di un valore pari a 17 mila fiorini (Pol, 1823, p. 79) comprendeva circa trecento manoscritti di autori classici (Aristotele, Cicerone, 
Euripide, Omero, Giovenale, Lucano, Ovidio, Plutarco, Seneca, Tolomeo, Terenzio e Vitruvio), seimila libri (di letteratura, storia, matematica, medicina, diritto e teologia) e anche un'imponente raccolta di quadri, statue, monete e minerali ${ }^{1}$. Secondo la volontà del fondatore, tutta la collezione doveva portare il suo nome, indipendentemente da quelli che ne sarebbero stati successivamente i beneficiari, e doveva essere interamente messa a disposizione del pubblico. Tuttavia, questo fu possibile soltanto nel 1661; dieci anni dopo, deposta presso l'Auditorium Theologicum della chiesa di Santa Elisabetta a Breslavia, fu irrobustita dalla donazione di uno degli insegnanti del Gymnasium Elisabethanum, Daniel Sartorius (1612-1671). Sartorius raccolse un'imponente collezione di musica a stampa pubblicata in quegli anni soprattutto presso le officine veneziane.

Poco prima aveva iniziato a raccogliere un repertorio di questo tipo un suo compagno di ginnasio, più anziano, Ambrosius Profius (1589-1661), organista della chiesa breslaviana di Santa Elisabetta. Dopo aver messo insieme un ingente patrimonio grazie alla vendita di vestiti, egli aveva comprato alcune decine delle edizioni musicali italiane più nuove e aveva finanziato la pubblicazione di alcune antologie, comprendenti opere di compositori dell'Italia settentrionale (Starke, 1902, pp. 210-213). Gran parte dei brani da lui pubblicati sono adattamenti di madrigali italiani ai quali erano stati abbinati testi in tedesco a tematica religiosa (Steude, 1992), rappresentando un esempio della pratica di contraffazione, molto comune a quel tempo. Il loro autore potrebbe essere stato lo stesso Profius, oppure i suoi collaboratori, che in alcune edizioni sono caratterizzati da monogrammi (Profius, 1627) ${ }^{2}$. Nella prefazione ad uno dei tomi Profius rivela di voler pubblicare questi brani a scopi didattici (Profius, 1646, libro Tenor, c. A3v), ma afferma anche di contare sul fatto che essi possano entrare nella pratica del repertorio

${ }^{1}$ Per un inventario storico della collezione può risultare utile il libretto dello spettacolo teatrale scritto da Gottlob Krantz e presentato a Breslavia nel 1698 nella forma di un dialogo scenico (Krantz, 1699).

2 Accanto alle contraffazioni di questa raccolta compaiono i seguenti monogrammi: J. E. G. S.; Dn D. G. M.; Dn. M. A. T.; Dn. J. P.; Dn. C. T.; Dn. I. F.; Dn. M. F.; Dn. M. G.; Dn. D. W.; Dn. D. I. S. p. m. e E. G.S. 
delle chiese. Proprio a tale scopo egli inserì nei madrigali di Claudio Monteverdi, Alessandro Grandi e Giovanni Rovetta parafrasi di citazioni bibliche. Aggiunse alle composizioni italiane già esistenti i nuovi testi letterari in modo tale da conservare l'impianto versificatorio dei madrigali e la persuasività dell'effetto retorico, caratteristica di questo genere. Il lavoro di Profius derivava dunque direttamente dagli ideali umanistici, così fortemente presenti nel programma di insegnamento del ginnasio elisabettiano.

Uno stretto collaboratore di Profius era Daniel Sartorius, che aveva ereditato dal suo collega più anziano l'interesse per la musica italiana del periodo e la passione per il collezionismo. Le stampe da lui raccolte si distinguevano stilisticamente dal repertorio che allora si eseguiva nella chiesa di Santa Elisabetta, dove fino alla metà del Seicento era predominante la musica della tradizione rinascimentale latina (Jeż, 2017a, p. 15). Sartorius ampliò la collezione di Profius di quasi quattrocento stampe con le opere più importanti della musica del primo barocco, composte soprattutto nella Repubblica di Venezia e stampate nelle officine della Serenissima (specialmente presso Alessandro e Giacomo Vincenti e Bartolomeo e Francesco Magni). Che il repertorio da lui collezionato venisse usato anche in pratica è testimoniato dalle annotazioni scritte sulle partiture dallo stesso Sartorius; in alcuni casi alle composizioni erano abbinati nuovi testi, scritti sotto il pentagramma oppure su fogli appositamente aggiunti ${ }^{3}$. Lo testimoniano anche le trascrizioni manoscritte delle composizioni stampate, in parte effettuate dall'insegnante di Santa Elisabetta e firmate col suo monogramma (D.S.), che integrano la collezione $e^{4}$. Molte testimonianze di un diretto impegno di Sartorius nella vita musicale di Breslavia si possono trovare nelle annotazioni del rettore del ginnasio elisabettano, Elias Maior (1587-1669; Jeż, 2017b, pp. 286-287). Ne risulta che Sartorius non solo partecipava all'esecuzione delle musiche nella scuola e nella chiesa, ma anche spro-

${ }^{3}$ Per esempio la contraffazione del madrigale di Giovanni Rovetta, Giovinetta fastosa, nella stampa Rovetta, 1636, libro S II/T conservato a Wrocław, Biblioteka Uniwersytecka 50752 Muz.

${ }^{4}$ Si tratta dei 37 manoscritti conservati oggi nella Staatsbibliothek zu Berlin Preußischer Kulturbesitz. Cf. Bohn, 1890, p. XII. 
nava i suoi allievi affinché cantassero e suonassero in cerimonie private, che solitamente si svolgevano cum vino et carminibus (Major, 1640, fol. 103r).

I diversi generi musicali che troviamo nelle stampe raccolte da Profius e Sartorius corrispondono alla variegata funzione della musica. Accanto alle raccolte liturgiche, che sono dominanti (alcune hanno titoli che si rifanno a termini concettuali, secondo la moda italiana: affetti (Strozzi, 1655), delizie (Milanuzzi, 1636), fiori (Tarditi, 1640), scherzi (Cozzolani, 1648), sentimenti (Legrenzi, 1660) o vaghezze (Gallerano, 1628), suggerendo la novità del linguaggio musicale nelle opere ivi contenute), appaiono qui anche composizioni sceniche (Monteverdi, 1607), raccolte di madrigali (Grandi, 1623) e di musica strumentale. I compositori della grande maggioranza delle musiche a stampa della collezione di Breslavia erano autori attivi a Venezia (Dario Castello, Francesco Cavalli, Alessandro Grandi, Giovanni Legrenzi, Biagio Marini, Claudio Monteverdi, Giovanni Antonio Rigatti) e nelle altre città della Repubblica: Bergamo (Giovanni Cavaccio, Francesco Rogantini, Pietro Andrea Ziani), Padova (Leandro Gallerano, Salvatore de Santa Maria, Simone Vesi), Udine (Orindio Bartolini, Pietro Gamberi, Luigi Pozzi), Verona (Nicolò Fontei, Biagio Gherardi, Francesco Rasi) e Vicenza (Gaspare Filippi, Amadio Freddi, Domenico Freschi, Carlo Grossi). Il territorio della Serenissima comprendeva anche i luoghi di residenza di potenti e nobili ai quali veniva dedicata la grande maggioranza dei libri della collezione qui studiata; lo stesso riguarda i conventi e le confraternite laiche che in quel periodo erano importanti mecenati della cultura musicale.

Ma quale significato aveva la collezione per la cultura della Breslavia del diciassettesimo secolo? Se vogliamo trattare questa particolare raccolta come una sorta di portatore di significato appropriato per un dato ambiente in un luogo e in un tempo preciso, la teoria dei semiofori presentata nelle pubblicazioni di Krzysztof Pomian sarà utile per rispondere a questa domanda (Pomian, 2006, p. 100 ss.). Il collezionismo intenzionale di musica a stampa adempie infatti ai criteri metodologici proposti dallo studioso per i semiofori: essi uniscono nella propria natura proprietà fisiche e semiotiche, dunque li si può studiare e confrontare sia 
come oggetti materiali sia come significati. Mentre la prima prospettiva descriverà le caratteristiche oggettive dei singoli tomi stampati in molte copie, la seconda porrà l'accento su caratteristiche specificatamente locali. In quest'ultima prospettiva, il paradosso della storia è che oltre il $25 \%$ del materiale raccolto consiste in esemplari unici, attualmente non conservati in nessuna altra biblioteca del mondo. Il fatto che più di cento pubblicazioni delle officine veneziane si sia conservato esclusivamente a Breslavia (Castello, 1629; Chinelli, 1637; Filago, 1642; Gibellini, 1655; Marini, 1629) può essere dunque considerato in quest'ottica semioticamente significativo.

Proseguendo sulla scia di Pomian (Pomian, 2006, p. 101), è impossibile non notare che le stampe di testi musicali di maestri italiani collezionate sull'Oder possono essere definite sia come testi di cultura sia come immagini di essa: come testi adattati alle specifiche condizioni della cultura locale (mediante la traduzione o la contraffazione e anche come immagini di un'altra cultura, portata dall'Italia e trattata come modello da imitare. La collezione breslaviana rivela l'orientamento particolarmente positivo dei suoi possessori verso il repertorio italiano contemporaneo, nonché l'ampio orizzonte estetico e l'indubbio gusto musicale degli stessi. Eppure, questa non era una qualità comune fra gli insegnanti di ginnasio dell'epoca! I materiali qui analizzati permettono anche di descrivere con precisione i tratti comuni del repertorio raccolto da Profius e Sartorius; entrambi lasciarono del resto alcune tracce di sé, ossia cinque manoscritti annessi alla collezione, scritti o riforniti da loro. Queste fonti rivelano non solo delle preferenze stilistiche dei collezionisti breslaviani, ma anche la loro competenze compositive nell'adattamento alla locale pratica compositiva. Detti manoscritti contengono fra l'altro copie di: composizioni strumentali di Adam Jarzębski (Jarzębski, 1627) - violinista alla corte di Sigismondo III Vasa a Varsavia; composizioni di Riccardo Rognoni dedicate a questo sovrano (Rognoni, 1626) e composizioni teatrali di Daniel Bollius, maestro di cappella e organista alla corte degli arcivescovi-elettori di Magonza (Bollius, 1623). Tutti i cinque manoscritti sono legati dallo stile musicale del primo barocco dell'Italia settentrionale e hanno in comune anche i legami culturali dei loro copisti con la città di Francoforte sul Meno, che era all'epoca 
il principale centro europeo per il commercio della musica a stampa (Epstein, 1924, p. 73).

La musica a stampa può essere del resto inserita nella categoria di quelle pubblicazioni che, nella riflessione di Pomian, rappresentano il più ovvio esempio di semiofori. Così come ogni altro libro, anche una stampa musicale "rimanda ad un lettore che è esterno rispetto ad essa e ad un significato invisibile che egli può ricavare dai segni che essa contiene" (Pomian, 2006, p. 121). L'opera musicale immortalata nel testo stampato può rivelarsi, anche grazie alla sua lettura, nella mente di chi la studia o nella pratica esecutiva di un dato ambiente: qui abbiamo a che fare con entrambe le forme. Anche se per parte della musica a stampa non c'era all'epoca occasione di essere utilizzata in questo modo (molti volumi della raccolta di Sartorius non riportano nessuna annotazione scritta, il che permette di avanzare una simile ipotesi), lo stesso fatto che dei collezionati libri fossero stati comprati e collocati su uno scaffale mostra indirettamente la funzione che il loro contenuto aveva per i collezionisti del diciassettesimo secolo. Per quanto le prime due funzioni impongano di trattare la collezione di musicalia come testi, è quest'ultima a collocarsi nella categoria che Pomian definisce pezzo da collezione, un oggetto che assume significato grazie all'esposizione e alla decontestualizzazione (ibid., pp. 124, 129). La prima di queste attività serve a circondare il semioforo della protezione bibliotecaria; la seconda - che nel nostro caso viene raggiunta tramite la contraffazione gli assicura una nuova funzione nella cultura locale.

Se accettiamo la tesi di Pomian, che postula la necessità di unire in una riflessione storico-culturale l'approccio ermeneutico e quello semiotico, occorre analizzare il significato della collezione breslaviana di $m u$ sicalia non solo dal punto di vista della genesi della collezione (che nel nostro caso può essere descritta semplicemente come storia della musica del Seicento italiano), ma soprattutto in riferimento alla sua ricezione, "infatti è nel corso della ricezione che gli utenti attualizzano le funzioni degli oggetti e ne costituiscono i significati" (ibid., p. 137). La ricezione del repertorio messo insieme da Profius e Sartorius è confermata dalla contraffazione dei madrigali approntata da loro stessi, dalle loro annotazioni manoscritte e dalle testimonianze a noi giunte riguardo il modo 
in cui la musica italiana più recente veniva eseguita in quel periodo, sia nelle scuole di Breslavia che nelle chiese. Ambedue le istituzioni - legate fra loro da un sistema culturale simbiotico - basavano all'epoca la propria formazione su una nuova forma di memoria collettiva, ridefinita rispettivamente dall'Umanesimo e dalla Riforma (Gancarczyk, 2011, pp. 203-204). Entrambe queste correnti di pensiero - che erano interdipendenti e si integravano a vicenda - portarono a una radicale ristrutturazione della memoria delle élite e giocarono un ruolo significativo nella formazione della loro nuova identità (Pomian, 2006, p. 162).

Il fatto che la stampa si sia rivelata il mezzo-chiave in questa nuova forma di memoria, nel contesto della collezione qui studiata, appare tutt'altro che casuale. Fu infatti la stampa a dare profondità al processo, iniziato dal Rinascimento, finalizzato a riportare in vita le vecchie abitudini, e al tempo stesso "tagliò i lacci che tenevano attaccati i testi alle istituzioni e li introdusse nella sfera pubblica" (ibid., p. 165). Canonizzando la superiorità dello scritto sul parlato, essa provocò un'interruzione della continuità culturale senza precedenti, sia nel dominio del modello educativo sia nell'autorevolezza del passato (Budzyński, 2003, p. 138). Poiché entrambi i fenomeni facevano da sfondo culturale alla ricezione dei materiali musicali della raccolta breslaviana, vale la pena di porsi questa domanda: potevano all'epoca essere considerati anch'essi come sintomi di fenomeni analoghi? Se guardiamo più da vicino il repertorio musicale raccolto da Sartorius, possiamo constatare senza difficoltà che esso rappresenta - nel contesto della pratica esecutiva vigente in quell'ambiente fino a quel momento - un elemento del tutto nuovo e culturalmente estraneo.

Non si tratta qui esclusivamente della provenienza cattolica delle stampe finite nell'ambiente della chiesa protestante, né del profilo del repertorio di importazione, inadeguato rispetto alla funzione che poteva avere in quell' ambito locale, bensì innanzitutto della sua stilistica musicale straordinariamente moderna, che rappresentò una delle cesure più importanti nella storia della musica. Tale cesura era legata all'apparizione di un nuovo stile in una musica che, in accordo agli ideali umanistici, doveva da quel momento in poi essere totalmente sottomessa alla parola, in modo da poter rendere, grazie ai suoni, gli affetti nascosti nelle 
parole (Palisca, 1968, p. 3). Questa nuova pratica musicale rappresentò una rottura senza precedenti con la precedente tradizione compositiva, nonostante fosse stata ispirata dal motto rinascimentale del ritorno alle fonti, e queste fonti illuminassero altrettanto bene sia l'Umanesimo che la Riforma. Tuttavia, l'idea stessa di riportare in auge i valori originali nella religione, nella scienza e nell'arte del suono, si legava ad uno sguardo critico nei confronti della cultura ereditata nella tradizione, una critica che i contemporanei consideravano non tanto come un'innovazione, quanto piuttosto come una resurrezione del passato che soltanto oggi, in una prospettiva storica, possiamo riconoscere come una forma di invented tradition (Hobsbawm \& Ranger, 1996).

\section{BIBLIOGRAFIA}

Adrio, A. (1962). Ambrosius Profe (1589-1661) als Herausgeber italienischer Musik seiner Zeit. In H. Hüschen (Ed.), Festschrift Karl Gustav Fellerer zum sechzigsten Geburtstag am 7. Juli 1962 (p. 20-27). Regensburg: Bosse.

Bauch, G. (1896). Beiträge zur Literaturgeschichte des schlesischen Humanismus. Zeitschrift des Vereins für Geschichte und Altherthum Schlesiens, 30, 127-164.

Bohn, E. (1890). Die musikalischen Handschriften des XVI. und XVII. Jahrhunderts in der Stadtbibiliothek zu Breslau. Breslau: Commissions-Verlag von Julius Hanauer.

Bollius, D. (1623). REPRESENTATIO | HARMONICA. | CONCEPTIONIS \& NATIVITATIS | S. JOANNIS BAPTISTA | INTER NATOS MVLIERVM MAXIMI | iuxta | SANCTVM | JESV CHRISTI | EVANGELIUM | Secundum Lucam | compofita | MODO PATHETICO SIVE RECITATIVO | distributa | IN DVOS ACTVS, ET SEX SCENAS | adiunctis | QUINQUE SYMPHONIIS | loco intermedij. | AVTORE | DANIELE BOLLIO (manoscritto). Staatsbibliothek zu Berlin - Preussischer Kulturbesitz, Slg. Bohn 129.

Budzyński, J. (2003). Józef Budzyński, Paideia humanistyczna czyli wychowanie do kultury: studium z dziejów klasycznej edukacji w gimnazjach XVI-XVIII wieku (na przyktadzie Śląska). Częstochowa: Wydawnictwo Wyższej Szkoły Pedagogicznej. 
Castello, D. (1629). SONATE CONCERTATE | In Stil Moderno, per Sonar nel Organo, | ouero Spineta con diuerfi Inftrumenti. | A 2. \& 3. Voci, | Con Baffo Continuo. | LIBRO PRIMO. | Con Priuilegio. | DI DARIO CASTELLO VENETIANO. | Capo di Compagnia de Mufichi [!] | d'Inftrumenti da fiato | In Venetia || STAMPA DEL GARDANO. | IN VENETIA M.DC.XXIX. || Appreffo Bartholomeo Magni.

Chinelli, G. (1637). IL PRIMO LIBRO | DI | MOTETTI $\mid$ A VOCE SOLA DI GIO. BATTISTA | CHINELLI | OPERA QVINTA. | Nuouamente compofta, \& data in luce | CON PRIVILEGIO. | ET DEDICATA | Alla Molto Illuftre, \& Molto Reuerenda Signora $\mid$ La Signora $\mid$ SVOR RAFFAELLA ALEOTTI | Organifta Eccellentiffima, \& Priora nel Monaftero | delle Monache di San VITO di Ferrara. || IN VENETIA, | Appreffo Alesfandro Vincenti. | MD CXXXVII.

Cozzolani, M. (1648). SCHERZI DI | SACRA $\mid$ MELODIA $\mid$ A VOCE SOLA | di Donna | CHIARA MARGARITA COZZOLANI | Monaca in Santa Radegonda di Milano. | DEDICATI | AL REVERENDISSIMO PADRE | DON | CLAVDIO BENEDETTI | DI VERONA | Prefidente Generale della Congregatione Caffinenfe. | OPERA TERZA Con Priuilegio. || IN

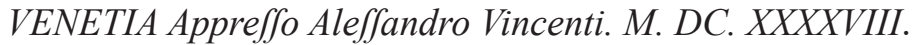

Filago, C. (1642). SACRI CONCERTI |A Voce fola. | Con la Partitura Per L'organo | DI | CARLO FILAGO | RODIGINO | Organifta Della Sereniffima Republica | di Venetia In S. Marco. | Opera Quarta. Con Licenza de Superiori. || IN VENETIA M DC XXXXII || Appreffo Bartolomeo Magni.

Gallerano, L. (1628). CVRIOSO MISTO $\mid$ DI VAGHEZZE $\mid$ MUSICALI $\mid$ COMPOSTO DA | LEANDRO GALLERANO | DA BRESCIA | ACCADEMICO OCCVLTO DELLO L'INVOLATO. | Maestro di Capella della Veneranda Arca del Glo-|rioso S. ANTONIO da Padoa. | VOCE SOLA. | OPERA XV. $\mid$ DEDICATO. | Al Molto Illustre Signor Antonio Megliara. | In Venetia, Appresso Alessandro Vincenti 1628.

Ganczarczyk, P. (2011). Muzyka wobec rewolucji druku. Przemiany w kulturze muzycznej XVI wieku. Toruń: Uniwersytet Mikołaja Kopernika w Toruniu.

Gibellini, N. (1655). MOTETTI $\mid$ A 2. 3. e 4 Voci $\mid$ DEL PADRE FRA NICOLA GIBELLINI | Da Norcia Agoftiniano | Maeftro di Capella in S. Stefano di Venetia. $\mid$ Libro Primo. Opera Seconda $\mid$ Dedicati $\mid$ AL MOLTO R. ${ }^{d o}$ PADRE MAESTRO | NICOLA ANGELVCCI | Da Norcia del medemo 
ordine. | [...] | Stampa del Gardano | IN VENETIA M DC LV Apreffo France fo Magni.

Grandi, A. (1623). MADRIGALI | CONCERTATI | A Due, Tre, \& Quattro, voci per cantar | e fonar nel Clauicembalo, Chitar-|rone, ò altro fimile ftromento. | DI ALESSANDRO GRANDI | Vice Maeftro di Capella della Sere-|nisfima Signoria di Venetia | In San Marco. | Nuouamente riftampati, \& corretti. | CON PRIVILEGIO. | LIBRO SECONDO |OPE-

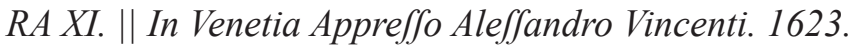

Hobsbawm, E.J., \& Ranger, T. (Eds.). (1996). The Invention of Tradition. Cambridge: Cambridge University Press.

Houszka, E. (1998). Prehistoria wrocławskich muzeów. In P. Łukaszewicz (Ed.), Muzea sztuki w dawnym Wrocławiu (pp. 11-26). Wrocław: Muzeum Narodowe we Wrocławiu.

Epstein, P. (1924). Die Frankfurter Kapellmusik zur Zeit J. A. Herbst's. Archiv für Musikwissenschaft, 6, 58-102.

Jarzębski, A. (1627). Adam Jarzębski, Canzoni e Concerti. I A due, Tre, e Quattro Voci $\mid$ Cum Ba/so Continuo | Di $\mid$ Adamo Harzebskÿ: | Polono. | Anno. | M.DC.XXVII (manoscritto). Staatsbibliothek zu Berlin - Preussischer Kulturbesitz, Slg. Bohn 111.

Jeż, T. (2017a). Danielis Sartorii Musicalia Wratislaviensia. Warszawa: Sub Lupa.

Jeż, T. (2017b). From a Musical Humanist's Diaries. The Schreibkalenders of Elias Maior (1588-1669) as a Source for the History of Wrocław Musical Culture, Odrodzenie i Reformacja w Polsce, LXI, 275-302.

Kolbuszewska, A. (2008). Katalog rękopisów muzycznych XVIII i XIX wieku Biblioteki Uniwersyteckiej we Wrocławiu. Wrocław: Wydawnictwo Uniwersytetu Wrocławskiego.

Krantz, G. (1699). Memorabilia | Bibliotheca $\mid$ Publica | Elisabetance | Wratislaviensis, $|\grave{a}|$ Fundatore Celeberrimo $\mid$ Rehdigeriance $\mid$ dictoe, | quce | A. O. R. M. DC. XCIIX. Die XV Maji, | In Actu Gymnico | à Studiosâ Gymnasii Elisabetani Juventute | exponi fecit. | Gottlob Krantz| Histor. Eloq. \& Phys. | Prof. \& Bibliothecarius. | Wratislavioe, | apud Johannem Georgium Steck, | M.DC. XCIX.

Legrenzi, G. (1660). SENTIMENTI $\mid$ DEVOTI $\mid$ Efpreffi con la Mufica di due, e tre voci | Da GIO: LEGRENZI Maeftro di Capella nell'Illuftriffima | accademia del Spirito Santo di Ferrara. | Dedicati | ALL'ILLVSTRISSIMO SIGNOR CONTE | GEROLAMO SIMONETTA | Libro Secondo 
| Opera Sefta. || IN VENETIA MDCLX. Apreffo Francefco Magni detto Gardano.

Major, E. (1640). Elias Major, Schreibkalender auffs Jahr nach Christi Geburt 1640 (manoscritto). Wrocław, Biblioteka Uniwersytecka, Oddział Rękopisów R 2339.

Marini, B. (1629). SONATE | SYMPHONIE | Canzoni, Pafs'emezzi, | Baletti, Corenti, | Gagliarde, | \& Retornelli, |A 1. 2. 3. 4. 5. \& 6. Voci, | Per ogni forte d'Inftrumenti. | Vn Capriccio per Sonar due Violini Quatro parti. Vn Ecco | per tre Violini, \& alcune Sonate Capricciofe per Sonar | due e tre parti con il Violino Solo, con altre | curiofe \& moderne inuentioni. Opera Ottaua | CON PRIVILEGIO. | DEL SIGNOR BIAGIO MARINI| Accademico Occulto Gentilhomo | è Maeftro della Mufica. | DEL SER. ${ }^{\text {mo }}$ SIG.r VOLFGANGO VILLELMO | Conte Palatino del Reno, Duca di Bauiera, | Giugliers, Cleues, \& Berg. \&c. || STAMPA DEL GARDANO | IN VENETIA M DC XXVIIII. \| Appreffo Bartolomeo Magni.

Milanuzzi, C. (1636). HORTVS SACER | DELICIARVM, | Ex quo Flores, \& Fructus per voces, tum fingulas, tum binas, | tum ternas ad auditum accomodati; colleguntur fuauiffimi | VNA CVM MISSA, DVABVS, AC LITANIIS | BEATAE MARIAE VIRGINIS | Tribus vocibus decantandis in Organo. | AVCTORE | F. CAROLO MILANVZIO | A SANCTA ANATHOLIA AVGVSTINIANO | MVSICAE PRAEFECTO CAMERINI | LIBER TERTIVS | Opus decimum nonum, nunc primum in lucem ęditum. | CVM PRIVILEGIO. || VENETIIS. Apud Alexandrum Vincentium. $M D C X X X V I$.

Monteverdi, C. (1607). L'ORFEO | FAVOLA $\mid$ IN MUSICA $\mid$ DA CLAVDIO MONTEVERDE | MAESTRO DI CAPELLA | DELLA SERENISS. REPVBLICA. | RAPPRESENTATA IN MANTOVA. | L'Anno 1607. Et nuovamente Riftampata. $\|$ IN VENETIA M DC X.V. \| Appreffo Ricciardo Amadino.

Palisca, C.V. (1968). Baroque Music. Englewood Cliffs, New Jersey: Prentice Hall.

Pol, N. (1823). Jahrbücher der Stadt Breslau von Nikolaus Pol. Zum erstenmale aus dessen eigener Handschrift herausgegeben von Dr. Johann Gustav Büsching, Königlicher Professor und Archivar und J. G. Kunisch. Band IV. Breslau: Gustav Kupfer.

Pomian, K. (2006). Historia: nauka wobec pamięci. Lublin: Wydawnictwo Uniwersytetu Marii Curie-Skłodowskiej. 
Profius, A. (1627). Extract oder Erster Theil auss dem Musicalischen Interim Ambrosii Profii, darinnen etlicher vornehmer und berühmter Autorum Madrigalien und anmutige Cantiones mit Deutschen Geistlichen und Politischen Texten (an statt der Italienischen) unterleget mit 3. 4. 5. 6. und 7. Stimmen, Allen Liebhabern der Edlen Musica, inn- und ausser der Kirchen jederzeit zu gebrauchen, zu gefallen publiciret. Wittenberg: J. Gorman.

Profius, A. (1646). Vierdter und letzter Theil $\mid$ Geistlicher $\mid$ CONCERTEN, Aus den berühmstenItaliänischen | und andern Authoribus, so theils mit andern, oder | auch noch mehren Texten beleget, und zum Lobe Gottes, | in öffentlichen Kirchen-Versammlungen zu gebrauchen, auch zu $\mid \mathrm{Ge}$ fallen allen Music-Liebhabern, | colligiret | und | publiciret | von | Ambrosio Profio Organ. zu S. Eli-|sabeth in Bresslaw |... Leipzig: Gedruckt bey Timotheo Ritzschen.

Rognoni, F. (1626). Selua de varii pasfaggi | secondo l'uso moderno, per cantare e suonare ogni $\mid$ sorte de stromenti, divisa in due Parti. Nella prima de quali $\mid$ si dimostra il modo di cantar polito, e con gratia, e la ma-|niera di portar le voce accentata, con tremoli, groppi, trilli, | esclamationi et pasfeggiare di grado in grado, salti di terza, quinta, | sesta, ottava, et cadenze finali per tutte le parti, con diversi altri | essempi et motetti passeggiati: cosa ancora utile à Suonatori | per imitare la voce humana. Nella seconda poi si tratta de | passagi difficili per gl'instromenti, del dar l'arcata, o lireggiare, | portar della lingua, diminuire di grado in grado, cadenze finali, | essempi con canti diminuiti, con la maniera di suonare alla $\mid$ bastarda. Milano, 1620 und 1626 (mit einer lateinischen Inschrift an den König Sigismund III in Polen) da | Francesco Rognone Taeggio (manoscritto). Staatsbibliothek zu Berlin - Preussischer Kulturbesitz, Slg. Bohn 113.

Rovetta, G. (1636). MADRIGALI CONCERTATI $\mid$ A. 2. 3. 4. \& vno a Sei voci, $\&$ due Violini $\mid$ Con vn Dialogo nel Fine, \& vna Cantata $\mid$ a voce Sola. | DI GIO. ROVETTA | Vice Maeftro di Capella della Serenisfima | Republica. | Libro Primo. Opera Seconda. | Novamente Riftampati. | Con Licenza de Superiori \& Priuilegio. | [...] | IN VENETIA M DC XXXVI. II Appreffo Bartolomeo Magni.

Sponheim, K.M. (1995). The Anthologies of Ambrosius Profe (1589-1661) and the Transmission of the Italian Music in Germany (Doctoral Dissertation). Yale University. 
Starke, R. (1902). Ambrosius Profe. Monatshefte für Musikgeschichte, 34/11, 189-196; 34/12, 199-215.

Steude, W. (1992). Wrocławski organista Ambrosius Profius (1589-1661) jako edytor i wydawca muzyki wokalnej XVII w. In A. Wolański, \& M. Zduniak (Eds.), Tradycje Ślaskiej Kultury Muzycznej, t. 6. Księga konferencji 5-7 IV 1990 (pp. 51-60). Wrocław: Akademia Muzyczna im. Karola Lipińskiego.

Strozzi, B. (1655). SACRI MVSICALI AFFETTI | DI BARBARA STROZZI | Confecrati | ALLA SERENISSIMA ANNA AVSTRIACA | ARCHIDVCHESSA D'INSPRVCH | Libro Primo. Opera Quinta. || Stampa del Gardano. IN VENETIA MDCLV Apreffo Francefco Magni.

Tarditi, O. (1640). CELESTI FIORI $\mid$ MVSICALI $\mid$ DI VARII CONCERTI SACRI $\mid$ A VOCE SOLA | Per Cantare Nell'Organo, Chitarrone, Arpa, Leuto, | Spinetta, \& Altro Stromento | COMMODI, ET FACILI PER OGNI CANTORE $\mid$ DI HORATIO TARDITI | ORGANISTA IN S. MICHELE DI MVRANO | LIBRO SECONDO. | OPERA OTTAVA | NOVAMENTE RI-

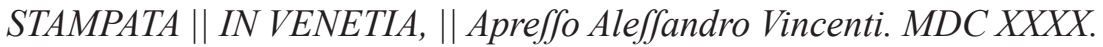
Visconti, G. (1637). CANZONI, | OVERO | SONATE CONCERTATE | PER CHIESA, | E CAMERA | A DVE, ET A TRE | DEL CAVALIERE | TARQUINIO MERVLA | LIBRO TERZO, OPERA DVODECIMA. | Dedicate all'Illuftriffimo Signore, | IL SIGNOR | GIO. BATTISTA VISCONTI. ||

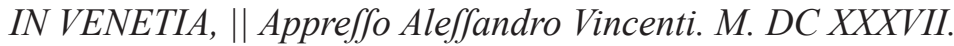

Wachler, A.W.J. (1828). Thomas Rehdiger und seine Büchersammlung in Breslau: Ein biographisch-literarischer Versuch. Breslau: Grüson.

Riassunto: Durante i suoi Wanderjahre, il patrizio di Breslavia Thomas Rehdiger (1540-1576) raccolse una ricca collezione di libri, manoscritti, monete, pietre preziose e opere d'arte. Nel suo testamento decise di destinare questa collezione, messa insieme soprattutto nei soggiorni a Padova, Bologna e Venezia, alla propria città natale, dando così inizio alla prima biblioteca pubblica della metropoli sull'Oder. Nel 1589 la Bibliotheca Rhedigeriana fu collocata nella sala dell'Auditorium Theologicum nella chiesa di Santa Elisabetta a Breslavia. Nel ginnasio che funzionava presso questa chiesa Rehdiger aveva studiato da giovane; quasi cento anni più tardi in quella scuola si trovò ad insegnare Daniel Sartorius (1612-1671), che raccolse un'imponente collezione di stampe musicali, pubblicate all'epoca nelle officine veneziane. Questa collezione, unica nel suo genere, conservata insieme alla Biblioteca Rhedigeriana, è stata successivamente fusa con il suo contenuto.

Quale significato ebbe questa collezione per la cultura di Breslavia nel Seicento? Fu messa insieme senza dubbio su ispirazione dell'umanesimo, coltivato fra le mura della scuola elisabettiana almeno fin dai tempi di Laurentius Corvinus. Doveva servire sicuramente alla didattica condotta nell'Elisabethanum e alla formazione dei gusti artistici degli allievi di quel centro, i quali nel corso delle lezioni o delle ricreazioni pomeridiane eseguivano la musica italiana più nuova. Si provò ad 
inserire questo repertorio, raccolto con accuratezza, anche durante la pratica liturgica della chiesa adiacente, sfruttando volentieri la tecnica allora in voga della contraffazione. La funzione semioforica della Biblioteca Sartoriana doveva tuttavia definire in primo luogo una nuova forma di memoria culturale per le élite di Breslavia e caratterizzarne il profilo originale dell'identità, un profilo orientato da quegli stessi ideali che guidavano i compositori dell'Italia settentrionale nel Seicento.

Parole chiave: Breslavia, Seicento, collezione musicale, chiesa di S. Elisabetta, Daniel Sartorius 